\title{
Investigation on Non-Segmentation Based Algorithms for Microvascu- lature Quantification in Retinal OCTA Images
}

\author{
Amr Abuzer ${ }^{1}$ \\ Ady Naber ${ }^{1}$ \\ Simon Hoffmann ${ }^{1}$ \\ Lucy Kessler ${ }^{2}$ \\ Ramin Khoramnia ${ }^{2}$ \\ Werner Nahm ${ }^{1}$ \\ 1 - Institute of Biomedical Engineering, Karlsruhe Institute of Technology, Karlsruhe, Germany, publications@ibt.kit.e- \\ $\mathrm{du}$ \\ 2 - Department of Ophthalmology, Heidelberg Klinikum, Heidelberg, Germany
}

\section{Introduction}

Optical Coherence Tomography Angiography (OCTA) is a promising imaging tool for diagnosis in ophthalmology. It allows the physician to monitor diseases affecting the vascular networks of the retina. Many studies have used quantification methods for the objective assessment of the OCTA data. However, investigations into the loss of information in OCTA images due to the segmentation of vessels have not been explored. This contribution uses Differential Box Counting (DBC), a texture-based quantification tool that does not require segmentation, to assess the loss of information compared to segmentation-based methods.

\section{Methods}

Three methods were investigated: Box Counting (BCD) and Information Dimension (ID) as segmentation-based methods and Differential Box Counting (DBC) as a texture-based method. The investigation was performed on a small data set composed of 9 in vivo human eyes. The data contained the Superficial Vascular Complex (SVC) and the Deep Vascular Complex (DVC). The performance of the methods in differentiating both layers, as well as the consistency of measurements on the same layer was tested. The performance parameters were the ICC and the Mann-Whitney U test. An ICC $>0.8$ and a p-value $<0.05$ was considered significant.

\section{Results}

The ICC values of the DBC showed 0,84 and 0,82 for the DVC and SVC, respectively. Comparatively, the ICC values for the ID and BCD showed 0,95 and 0,91 for the DVC and SVC, respectively. The p-values of the DBC between SVC and DVC were $<0.01$. The p-values of the BCD and ID between SVC and DVC were $<0.001$.

\section{Conclusion}

The DBC is less statistically significant than the segmentation-based BCD and ID. It is thought that the DBC is more sensitive to intensity variations in the OCTA images compared to segmentation-based quantifications. Although errors exist in the segmented data due to the variance in OCTA images, the measurement error in the DBC diverges quicker than the measurement error of the segmentation-based parameters, as the DBC takes the intensity of the pixels into account, whereas segmentation-based parameters are only concerned with whether a pixel is part of a vessel or not. Similarly, motion artefacts and projection artefacts with higher intensity values than their neighbouring pixels also point to a higher measurement error in the DBC compared to the segmentation-based ID and BCD. 


\section{Laser sound in water: acoustic signals generated by nanosecond laser}

Liza Lengert (Industrial and Biomedical Optics, Laser Zentrum Hannover e. V.), Hannover, Germany, 1.lengert@1zh.de Stefan Kalies (Institute of Quantum Optics, Leibniz University Hannover), Hannover, Germany, kalies@iqo.uni-hannover.de

Sonja Johannsmeier (Industrial and Biomedical Optics, Laser Zentrum Hannover e.V.), Hannover, Germany, s.johannsmeier@1zh.de

Tammo Ripken (Industrial and Biomedical Optics, Laser Zentrum Hannover e.V.), Hannover, Germany, t.ripken@1zh.de Hannes Maier (Clinic for Laryngology, Rhinology and Otology, Hannover Medical School), Hannover, Germany, maier.hannes@mh-hannover.de

Thomas Lenarz (Clinic for Laryngology, Rhinology and Otology, Hannover Medical School), Hannover, Germany, lenarz.thomas@mh-hannover.de

Alexander Heisterkamp (Institute of Quantum Optics, Leibniz University Hannover), Hannover, Germany, heisterkamp@iqo.uni-hannover.de, and Industrial and Biomedical Optics, Laser Zentrum Hannover e.V.

\section{Introduction}

In the context of developing alternatives for electrical stimulation in the cochlea, optoacoustic stimulation might offer advantages such as a higher frequency discrimination. We established an optical setup that enabled us to trigger acoustic cavitation events in water. It is based on the generation of a laser induced optical breakdown via the application of short laser pulses. While the general physics of laser induced breakdown are well-understood, it is still unclear to which extent its accompanying acoustic events can be combined to generate sound perception. This work aims to proof the concept of generating audible tones in water as a model for the perilymph inside the cochlea.

\section{Methods}

Our laser system consists of a solid-state pulsed laser with variable repetition rates. The laser pulses were focused into a water-filled cube using an objective. With our setup, single optical breakdown events can be triggered as well as arbitrary pulse sequences. Acoustic measurements were conducted with a hydrophone.

\section{Results}

The acoustic measurements showed single acoustic events corresponding to optical breakdown generated by single laser pulses. Pulse trains of different frequencies were applied, although the maximum frequency was limited by the kinetics of the optoacoustic events.

\section{Conclusion}

This work presents the experimental setup for optoacoustic sound-generation in water by triggered nanosecond laser pulses for sound generated by optical breakdown.

Acknowledgement: This work was supported by the German Research Foundation, Germany Clusters of Excellence Hearing4all (EXC 2177). 


\section{OCT visualization of ocular opacities for laser-based treatment of eye floaters}

Jaryi Lippek, ${ }^{1}$ J.Lippek@1zh.de;

Piet Dyrøy, ${ }^{1}$ P.Dyroey@1zh.de

Miroslav Zabic, ${ }^{1,2,3}$ M.Zabic@1zh.de

Dag Heinemann, 2, 3, 4 dag.heinemann@hot.uni-hannover.de

Sonja Johannsmeier, ${ }^{1}$ S.Johannsmeier@1zh.de

Tammo Ripken, ${ }^{1}$ T.Ripken@1zh.de

${ }^{1}$ Industrial and Biomedical Optics Department, Laser Zentrum Hannover e.V., Hannover, Germany

${ }^{2}$ Hannover Centre for Optical Technologies, Gottfried Wilhelm Leibniz University of Hannover, Hannover, Germany

${ }^{3}$ Institute of Horticultural Production Systems, Gottfried Wilhelm Leibniz University Hannover, Hannover, Germany

${ }^{4}$ Cluster of Excellence PhoenixD (Photonics, Optics, and Engineering - Innovation Across Disciplines), Hannover, Germany

\section{Introduction}

Dense mobile opacities in the vitreous body of the eye cast shadows on the retina and lead to the visible phenomenon often described as floaters. These opacities are often related to the ageing process of the eye and most people notice these floaters at least once in their life. Since most people live with eye floaters without limitations, there is an uncared group of patients, which suffer from more severe symptomatic floaters, resulting in visual impairments and a reduction of quality of life. Established surgical therapies are either highly invasive or possess a questionable success rate. In a new therapeutic approach, we aim at the development of safe laser vitreolysis by using optical coherence tomography (OCT) for a computer-guided procedure and femtosecond laser pulses for a reduction of the applied pulse energy. Here, we present a setup for OCT-based guidance for patient-specific therapy.

\section{Methods}

A Swept Source OCT setup was realized to visualize model floaters in the volume of explanted porcine eyes. A custom software segments the floaters from the rest of the volume and estimates the distance to the retina. It also provides coordinates for the femtosecond laser amplification.

\section{Results}

We can successfully visualize model floaters inside a volume of an artificial eye model with our OCT setup. The software can segment floaters, displays them in a 3D model and provides coordinates for controlling the treatment laser. Additionally, we can measure the safety distance to the retina to prohibit a laser pulse too close to vulnerable tissue.

\section{Conclusion}

Computer guided surgical procedures is a growing field in ophthalmology and requires a high level of precision in technical aspects. Automated visualization of symptomatic floaters may enable a safer procedure for treatment of this underrated syndrome.

IGF- Vorhaben Nr: 21011 N; Feinmechanik, Optik und Medizintechnik; Funded by Federal Ministry for Economic Affairs and Energy based on a resolution of German Bundestag 


\title{
Patient specific analysis of the extracellular matrix of triple negativ breast cancer
}

\author{
Hannes Kamin. ${ }^{1}$ h.kamin@.zh.de \\ Lena Nolte, ${ }^{1}$ 1.nolte@1zh.de \\ Jochen Maurer, ${ }^{2}$ jmaurer@ukaachen.de \\ Andreas Bleilevens, ${ }^{2}$ ableilevens@ukaachen.de \\ Elmar Stickeler, ${ }^{2}$ gynaekologie@ukaachen.de \\ Dag Heinemann 1,4,5 dag.heinemann@hot.uni-hannover.de \\ Sonja Johannsmeier ${ }^{1,3}$ s.johannsmeier@lzh.de \\ Tammo Ripken ${ }^{1,3}$ t.ripken@1zh.de
}

\begin{abstract}
${ }^{1}$ Industrial and Biomedical Optics Department, Laser Zentrum Hannover e.V., Hannover, Germany
${ }^{2}$ Clinic for Gynecology and Obstetrics, University Hospital Aachen, Germany

${ }^{3}$ Lower Saxony Centre for Biomedical Engineering, Implant Research and Development, Hannover Germany

${ }^{4}$ Department of Phytophotonics, Institute of Horticultural Production Systems, Leibniz University Hannover, Germany

${ }^{5}$ Hannover Centre for Optical Technologies (HOT), Leibniz University Hannover, Germany
\end{abstract}

\section{Introduction}

The triple negative breast cancer is an aggressive subtype characterised by the lack specific receptors which have been historically targeted for treatment. Therefore the identification of new targets or therapies as well as analysis of the cancer structure providing information about relapse rate or benefit of chemotherapy are of great importance.

\section{Methods}

Prior to imaging samples were optically cleared and fixed using a resin-based curing method (CRISTAL). Different samples of triple-negative breast cancer were imaged using both Scanning Laser Optical Tomography (SLOT) and multiphoton microscopy (MPM) enabling the analysis of the collagen network architecture on different scales. Second Harmonic Genereation (SHG) was used as an inherent contrast mechanism showing the generell structure of collagen and complemented by antibody staining to allow specific analysis of Collagen I. The image stacks were postprocessed using Hessian based structure filters and segmented by a Random Forest algorithm. Eventually the collagen structures were investigated by a custom fiber extracting algorithm and visualised depending on their orientation.

\section{Results}

The CRISTAL process enables sequential imaging of samples in different setups maintaining the fluorescence for several weeks. Imaging based on SLOT and MPM using the complementary contrast mechanisms fluorescence and SHG revealed different collagen structures on macroscopic and microscopic scales. Sample based postprocessing and segmentation and subsequent extraction of the collagen fiber network highlighted the singularity of each sample. The complete workflow from sample preparation was performed.

\section{Conclusion}

Samples from diffenent patients were analysed using the proposed methods and thus the diverse threedimensional fiber formations in the microscopic to mesoscopic range were compared. The techiques established here might contribute to patient based medicine in breast cancer by analysing the individual cancerous collagen network.

This work was funded by the Federal Ministry of Education and Research, Germany, Grant no. 031L0146C. The authors declare that there is no conflict of interest. 


\section{Laplace-Beltrami Refined Shape Regression Applied to Neck Recon- struction for Craniosynostosis Patients}

Matthias Schaufelberger, Karlsruhe Institute of Technology, Institute of Biomedical Engineering, Karlsruhe, Germany, matthias.schaufelberger@kit.edu

Reinald Kühle, Clinic of Oral and Maxillofacial Surgery, Heidelberg University Hospital, Heidelberg,

Germany, reinald.kuehle@med.uni-heidelberg.de

Frederic Weichel, Clinic of Oral and Maxillofacial Surgery, Heidelberg University Hospital,

Heidelberg, Germany, frederic.weichel@med.uni-heidelberg.de

Andreas Wachter, Karlsruhe Institute of Technology, Institute of Biomedical Engineering, Karlsruhe, Germany, andreas.wachter@kit.edu

Niclas Hagen, Institute of Medical Informatics, Heidelberg University Hospital,

Heidelberg, Germany, niclas.hagen@med.uni-heidelberg.de

Friedemann Ringwald, Institute of Medical Informatics, Heidelberg University Hospital,

Heidelberg, Germany, friedemann.ringwald@med.uni-heidelberg.de

Urs Eisenmann, Institute of Medical Informatics, Heidelberg University Hospital,

Heidelberg, Germany, urs.eisenmann@med.uni-heidelberg.de

Christian Freudlsperger, Clinic of Oral and Maxillofacial Surgery, Heidelberg University Hospital,

Heidelberg, Germany, christian.freudlsperger@med.uni-heidelberg.de

Werner Nahm, Institute of Biomedical Engineering Karlsruhe Institute of Technology, Karlsruhe, Germany, werner.nahm@kit.edu

\section{Introduction}

This contribution is part of a project concerning the creation of an artificial dataset comprising 3D head scans of craniosynostosis patients for a deep-learning-based classification. To conform to real data, both head and neck are required in the 3D scans. However, during patient recording, the neck is often covered by medical staff. Simply pasting an arbitrary neck leaves large gaps in the 3D mesh. We therefore use a publicly available statistical shape model (SSM) for neck reconstruction. However, most SSMs of the head are constructed using healthy subjects, so the full head reconstruction loses the craniosynostosis-specific head shape. We propose a method to recover the neck while keeping the pathological head shape intact.

\section{Methods}

We propose a Laplace-Beltrami-based refinement step to deform the posterior mean shape of the full head model towards the pathological head. The artificial neck is created using the publicly available Liverpool-York-Model. We apply our method to construct artificial necks for head scans of 50 scaphocephaly patients.

\section{Results}

Our method reduces mean vertex correspondence error by approximately $1.3 \mathrm{~mm}$ compared to the ordinary posterior mean shape, preserves the pathological head shape, and creates a continuous transition between neck and head.

\section{Conclusion}

The presented method showed good results for reconstructing a plausible neck to craniosynostosis patients. Easily generalized it might also be applicable to other pathological shapes. 


\section{Technical light-field setup for 3D imaging of the human nerve head validated with an eye model}

Stefan Schramm, Institute of Biomedical Engineering and Informatics, Faculty of Computer Sciences and Automation, Technische Universität Ilmenau, Ilmenau, Germany, stefan.schramm@tu-ilmenau.de Alexander Dietzel, Institute of Biomedical Engineering and Informatics, Faculty of Computer Sciences and Automation, Technische Universität Ilmenau, Ilmenau, Germany, alexander.dietzel@tu-ilmenau.de

Maren-Christina Blum, Institute of Biomedical Engineering and Informatics, Faculty of Computer Sciences and Automation, Technische Universität Ilmenau, Ilmenau, Germany, maren.blum@tu-ilmenau.de

Dietmar Link, Institute of Biomedical Engineering and Informatics, Faculty of Computer Sciences and Automation, Technische Universität Ilmenau, Ilmenau, Germany, dietmar.link@tu-ilmenau.de

Sascha Klee, Department of General Health Studies, Division Biostatistics and Data Science, Karl Landsteiner University of Health Sciences, Krems an der Donau, Austria, sascha.klee@kl.ac.at; Institute of Biomedical Engineering and Informatics, Faculty of Computer Sciences and Automation, Technische Universität Ilmenau, Ilmenau, Germany, sascha.klee@tu-ilmenau.de

\section{Introduction}

With the new technology of 3D light field (LF) imaging, fundus photography can be expanded to provide depth information. This increases the diagnostic possibilities and additionally improves image quality by digitally refocusing. To provide depth information in the human optic nerve head such as in glaucoma diagnostics, a mydriatic fundus camera was upgraded with an LF imager. The aim of the study presented here was the validation of the technical setup and resulting depth estimations with an appropriate eye model.

\section{Methods}

The technical setup consisted of a mydriatic fundus camera (FF450, Carl Zeiss Meditec AG, Jena, Germany) and an LF imager (R12, Raytrix GmbH, Kiel, Germany). The field of view was set to $30^{\circ}$. The eye model ( $24.25 \mathrm{~mm}$ total length) consisted of a two-lens optical system and interchangeable fundus models with papilla excavations from 0.2 to $1 \mathrm{~mm}$ in steps of $0.2 \mathrm{~mm}$. They were coated with red acrylic lacquer and vessels were drawn with a thin brush. 15 images were taken for each papilla depth illuminated with green light (wavelength $520 \mathrm{~nm} \pm 20 \mathrm{~nm}$ ). Papilla depth was measured from the papilla ground to the surrounding flat image region. All 15 measurements for each papilla depth were averaged and compared to the printed depth.

\section{Results}

It was possible to perform 3D fundus imaging in an eye model by means of a novel LF-based optical setup. All LF images could be digitally refocused subsequently. Depth estimation in the eye model was successfully performed over a $30^{\circ}$ field of view. The measured virtual depth and the printed model papilla depth is linear correlated.

\section{Conclusion}

The presented LF setup allowed high-quality 3D one-shot imaging and depth estimation of the optic nerve head in an eye model. 


\section{Automatic Hypercube Acquisition with high spatial and spectral resolution using a HSI Linescan Camera}

Robin Pape, Research Center for BioMedical Technology, University of Applied Sciences and Arts, Dortmund, Germany, robin.pape001@stud.fh-dortmund.de

Stefan Patzke, Research Center for BioMedical Technology, University of Applied Sciences and Arts, Dortmund, Germany, stefan.patzke@fh-dortmund.de

Jörg Thiem, Research Center for BioMedical Technology, University of Applied Sciences and Arts, Dortmund, Germany, joerg.thiem@fh-dortmund.de

\section{Introduction}

RGB-cameras capture the red, green and blue parts of visible light. Hyperspectral imaging (HSI) cameras have much higher spectral resolution, making them useful for medical applications such as tissue analysis for cancer detection. Pushbroom HSI-cameras have a high spectral resolution. However, it is necessary to scan the subject under examination and stitch the images afterwards. If the distance between camera and subject is known, one can calculate the scanning velocity to accomplish the stitching easily. This contribution proposes a self-contained, portable environment for taking hyperspectral images using a feature-based algorithm to stitch the images automatically, even if the distance is not known exactly or the velocity cannot be applied perfectly.

\section{Methods}

Inside a light-protected box, the subject is placed on a linear guide unit. The HSI-camera is mounted facing downward to the subject. The camera uses an imec LS150 linescan sensor with ARM/DSP embedded processor which operates in the range of $450-970 \mathrm{~nm}$ with a resolution of $2048 \times 5$ pixels per filter wavelength and 150 filterbands. The linear guide unit moves the subject while capturing the subject in every filterband. The stitching process matches image features between subsequent images, giving the displacement used for accurate alignment.

\section{Results}

This contribution shows that it is possible to use automatic feature matching to efficiently automate the stitching process of hyperspectral images. Instead of relying on an even and exact movement of the linear guide unit, the displacement is directly determined, making this solution more robust and easier to use.

\section{Conclusion}

As the complete assembly is contained in a relatively compact box and an ordinary laptop is sufficient to control every part of it and generate the images, the system is portable enough to be used in the field, bringing the benefits of hyperspectral imaging to new use cases, e.g. the acquisition in pathologic institutes. 


\section{Towards a new surgical system: parameter study on laser-based bone cement removal}

Björn Spoida, ${ }^{1}$ b.spoida@1zh.de

Dag Heinemann, 2,3,4 dag.heinemann@hot.uni-hannover.de,

Malte Ohlmeier, ${ }^{5}$ malte.ohlmeier@helios-gesundheit.de

Sonja Johannsmeier, ${ }^{1}$ s.johannsmeier@1zh.de

Tammo Ripken, ${ }^{1}$ t.ripken@1zh.de

${ }^{1}$ Industrial and Biomedical Optics Department, Laser Zentrum Hannover e.V., Hannover, Germany.

${ }^{2}$ Hannover Centre for Optical Technologies, Gottfried Wilhelm Leibniz University of Hannover, Hannover, Germany.

${ }^{3}$ Institute of Horticultural Production Systems, Gottfried Wilhelm Leibniz University Hannover, Hannover, Germany.

${ }^{4}$ Cluster of Excellence PhoenixD (Photonics, Optics, and Engineering - Innovation Across Disciplines), Hannover, Germany.

${ }^{5}$ Department of Orthopaedic Surgery, Helios ENDO-Klinik, Hamburg, Germany.

\section{INTRODUCTION:}

The medical gold standard for cemented hip revision surgery is currently mechanical removal. The considerable number of cases, resulting from increasing life expectancy and an implant lifetime of 10-15 years, legitimate the development of a system for gentle, patient-friendly bone cement removal. For this we investigate the laser-based cement ablation to facilitate its extraction in revision surgery.

\section{METHODS:}

Initially, a spectral analysis was executed to scrutinize laser-relevant properties of bone and bone cement. The analysis included newly produced samples and samples which had aged in the human body. Based on this, a laser ablation process was developed in an in vitro parameter study. The parameters (power, focal diameter, irradiation time, cooling) were investigated as well as the environmental conditions and boundary conditions prevailing in the processing area. For further information on heat generation and heat-affected zone, a finite element simulation will be performed.

\section{RESULTS:}

The optical analysis of the materials revealed a favorable ablation window at the wavelength of $2 \mu \mathrm{m}$. A wavelength range that can be used for selective illumination in image-guided surgery has also been identified, which extends the discriminability of the materials at the interface. The parameter study allowed for a precise evaluation to maximize the material removal rate and monitor the changes in temperature evolution during the ablation process. An ablation rate of up to $6 \mathrm{mg} / \mathrm{s}$ was realized while heat development was limited to $<47^{\circ} \mathrm{C}$. The simulation validated the temperature data. The simulation suggests that the heat development is related to the irradiated area of the cement.

\section{CONCLUSION:}

The results obtained from the measurements and simulations are a promising basis for the subsequent development of a demonstrator system. The requirements will be identified with a clinical partner and the resulting demonstrator will be tested and optimized under realistic conditions, taking a significant step towards precise and patientfriendly removal of bone cement.

This work was funded by "ForTra gGmbh für Forschungstransfer der Else Kröner-Fresenius-Stiftung“. 